\title{
openheart Prehospital opioid dose and myocardial injury in patients with ST elevation myocardial infarction
}

\author{
Himawan Fernando (D) , 1,2,3 Ziad Nehme, ${ }^{4,5}$ Karlheinz Peter, ${ }^{1,3}$ Stephen Bernard, ${ }^{5,6}$ \\ Michael Stephenson, ${ }^{5}$ Janet Bray, ${ }^{4}$ Peter Cameron, ${ }^{4}$ Andris Ellims, ${ }^{1}$ Andrew Taylor, ${ }^{1}$ \\ David M Kaye, ${ }^{1,7}$ Karen Smith, ${ }^{4,5}$ Dion Stub (D) , ${ }^{1,4,7}$ for the AVOID investigators
}

\begin{abstract}
- Additional material is published online only. To view please visit the journal online (http://dx.doi.org/10.1136/ openhrt-2020-001307).
\end{abstract}

To cite: Fernando H, Nehme Z, Peter K, et al. Prehospital opioid dose and myocardial injury in patients with ST elevation myocardial infarction. Open Heart 2020;7:e001307. doi:10.1136/

openhrt-2020-001307

Received 9 April 2020 Revised 18 May 2020 Accepted 27 May 2020
Check for updates

(c) Author(s) (or their employer(s)) 2020. Re-use permitted under CC BY-NC. No commercial re-use. See rights and permissions. Published by BMJ.

For numbered affiliations see end of article.

Correspondence to Dr Dion Stub; d.stub@alfred. org.au

\section{ABSTRACT}

Objective To characterise the relationship between opioid dose and myocardial infarct size in patients with ST elevation myocardial infarction (STEMI).

Methods Patients given opioid treatment by emergency medical services with confirmed STEMI were included in this secondary, retrospective cohort analysis of the Air versus 0xygen in Myocardial Infarction (AVOID) study. Patients with cardiogenic shock were excluded. The primary endpoint was comparison of cardiac biomarkers as a measure of infarct size based on opioid dose (low $\leq 8.75 \mathrm{mg}$, intermediate $8.76-15 \mathrm{mg}$ and high $>15 \mathrm{mg}$ of intravenous morphine equivalent dose).

Results 422 patients were included in the analysis. There was a significantly higher proportion of patients with Thrombolysis in Myocardial Infarction (TIMI) 0 or 1 flow pre-percutaneous coronary intervention (PCI) $(94 \%$ vs $81 \%, p=0.005)$ and greater use of thrombus aspiration catheters $(59 \%$ vs $30 \%, p<0.001)$ in the high compared with low-dose opioid group. After adjustment for potential confounders, every $1 \mathrm{mg}$ of intravenous morphine equivalent dose was associated with a $1.4 \%(95 \% \mathrm{Cl}$ $0.2 \%, 2.7 \%, p=0.028$ ) increase in peak creatine kinase; however, this was no longer significant after adjustment for TIMI flow pre-PCI.

Conclusions Our study suggests no benefit of higher opioid dose and a dose-dependent signal between opioid dose and increased myocardial infarct size. Prospective randomised controlled trials are required to establish causality given that this may also be explained by patients with a greater ischaemic burden requiring higher opioid doses due to more severe pain. Future research also needs to focus on strategies to mitigate the opioid-P2Y12 inhibitor interaction and non-opioid analgesia to treat ischaemic chest pain.

\section{INTRODUCTION}

While medical and interventional therapy has undergone rapid advancement in the treatment of acute coronary syndrome, treatment of the pain associated with myocardial infarction has remained relatively unchanged for over 50 years. Opioids remain the analgesic agent of choice at least partly due to early studies suggesting beneficial haemodynamic

\section{Key questions}

What is already known about this subject?

- Opioid analgesia commonly given to patients with myocardial infarction to treat ischaemic chest pain delays the intestinal absorption of P2Y12 inhibitors and this may lead to adverse cardiovascular outcomes.

What does this study add?

- There was no association between higher opioid doses and benefit in myocardial infarction. Instead, higher doses of opioids were correlated with greater infarct size based on cardiac biomarker release suggesting a dose-dependent relationship.

How might this impact on clinical practice?

- While prospective randomised clinical trials are required to establish causality between opioid dose and myocardial infarct size, given the growing body of evidence relating to the opioid-P2Y12 inhibitor interaction, investigation of alternative analgesic agents that do not interact with P2Y12 inhibitors or strategies to mitigate this interaction warrant further study to guide future application in clinical practice.

effects through reduced pain-related sympathetic stimulation, venodilatory and vasodilatory effects. ${ }^{1}$ Despite this, the clinical benefit of using opioids has never been evaluated in a prospective randomised study. In contrast, analysis of the Can Rapid Risk Stratification of Unstable Angina Patients Suppress Adverse Outcomes with Early Implementation of the ACC/AHA Guidelines (CRUSADE) registry first raised concerns regarding an interaction between opioid analgesia and oral P2Y12 inhibitor therapy, ${ }^{2}$ which was associated with an increased risk of adverse cardiovascular events in patients presenting with non-ST elevation myocardial infarction (NSTEMI). Following this, biochemical studies suggested that opioids reduced the bioavailability and antiplatelet activity of P2Y12 inhibitors. The proposed mechanism is opioid-induced 
gastroparesis leading to impaired gastrointestinal absorption of oral P2Y12 inhibitors. It is currently unclear from available observational clinical data whether this biochemical interaction leads to worse clinical outcomes due to conflicting results. ${ }^{2-5} \mathrm{It}$ is also unclear whether the haemodynamic benefits of opioids may lead to improved outcomes in patients receiving higher opioid doses.

Our group demonstrated that incremental supplemental oxygen was correlated with increased myocardial injury following ST elevation myocardial infarction (STEMI) ${ }^{6}$ This study aims to evaluate whether there is a relationship between the amount of opioids administered and biochemical, interventional and clinical outcomes after STEMI.

\section{METHODS}

\section{Study design}

This study is an exploratory, secondary observational analysis of the Air versus Oxygen in Myocardial Infarction (AVOID) trial. A detailed description of the AVOID study design and results has been previously published (NCT 01272713) ${ }^{78}$ Briefly, this was a prospective, multicentre, randomised controlled trial enrolling 638 patients with suspected STEMI between October 2011 and July 2014 transferred to 9 percutaneous coronary intervention (PCI) capable hospitals in Melbourne, Australia. The original study was approved by ethics committees at each participating hospital with delayed written informed consent from the participant or next of kin obtained as soon as patients were stabilised in hospital.

The current analysis was undertaken without direct patient involvement. For this analysis, patients were not invited to comment on the study design, assist in development of outcomes, discussion of results or in preparation of the manuscript.

\section{Participants}

Inclusion criteria for the AVOID study were patients 18 years or older with chest pain symptoms for $<12$ hours prior and a 12-lead ECG consistent with ST elevation. Exclusion criteria included hypoxaemia on room air $\left(\mathrm{SpO}_{2}<94 \%\right)$, oxygen administration prior to randomisation, altered conscious state or transport to a nonparticipating hospital.

Opioid administration was guided by current Ambulance Victoria guidelines for management of ischaemic chest pain. The guideline recommended up to $5 \mathrm{mg}$ morphine or $50 \mu \mathrm{g}$ fentanyl intravenously every $5 \mathrm{~min}$ as required. Patients could also receive up to $200 \mu \mathrm{g}$ of intranasal fentanyl every $5 \mathrm{~min}$ if intravenous access was not available. For this analysis, patients with cardiogenic shock and patients not receiving opioids were excluded.

\section{Study outcomes}

The AVOID study used highly correlated co-primary endpoints of peak troponin I (cTnI) and creatine kinase (CK) as biomarkers of myocardial injury. In addition to this, the area under the curve of cTnI and CK over the first 72 hours was measured. For this, blood sampling was performed 6 hourly for the first 24 hours then 12 hourly until 72 hours. Other secondary endpoints included ST-segment resolution, mortality and major adverse cardiac events at hospital discharge and 6 months. Cardiac MRI was also performed at 6 months in a subset of 139 patients to measure infarct size.

The current analysis aimed to evaluate the relationship between opioid dose and CK and cTnI as biomarkers for infarct size. Additionally, the impact of opioid dose on cardiac MRI-derived infarct size, procedural and clinical outcomes was sought. The initial analysis was performed on 439 patients who had a diagnosis of STEMI confirmed by coronary angiography. There was a very high rate of cardiogenic shock $(47 \%)$ in the no opioid group suggesting that these patients were not treated with opioids as they were critically unwell at presentation. Therefore, for this analysis, patients given no opioid medications $(n=17)$ were excluded from the analysis (see online supplementary figure 1). As such, 422 patients were included in the final analysis. Fentanyl dose (intravenous or intranasal) was converted into equivalent morphine dose by multiplying total dose by 100. If fentanyl and morphine were both used, then total fentanyl dose was converted to equivalent morphine dose and added to the total morphine dose. This provided a total opioid dose for each patient. Opioid total dose was stratified as low if less than or equal to $8.75 \mathrm{mg}$ of intravenous morphine equivalent, intermediate if between $8.76 \mathrm{mg}$ and $15 \mathrm{mg}$ intravenous and high if greater than $15 \mathrm{mg}$. These specific cut-offs were chosen as they approximated equal tertiles for analysis.

\section{Statistical analysis}

All statistical analysis was performed using SPSS V.22 (IBM).

Variables approximating a normal distribution were summarised as mean $\pm \mathrm{SD}$ and groups were compared using analysis of variance. Non-normally distributed variables were summarised as median and third quartiles (Q1, Q3) and compared using Wilcoxon rank-sum test. Binomial variables were expressed as proportions and $95 \%$ confidence intervals (CIs and compared using $\chi^{2}$ tests.

Trapezoidal integration was used to estimate total $\mathrm{cTnI}$ and $\mathrm{CK}$ release during the first 72 hours $\left(\mathrm{AUC}_{72}\right)$. Missing biomarker assays were replaced with multiple imputation using the Markov Chain Monte Carlo method. ${ }^{9} 10$ The adjusted effect of opioid dose on biomarkers of myocardial injury was assessed using linear regression. The model adjusted for age, sex, diabetes, dyslipidaemia, hypertension, smoking, symptom to intervention time, TIMI flow pre-PCI and left anterior descending (LAD) coronary artery as the culprit vessel in STEMI. A log transformation of the biomarker and infarct size based on cardiac MRI data significantly improved the normality of residuals. Comparison of the treatment effect was made after back transformation which then represented the percentage 
Table 1 Baseline characteristics

\begin{tabular}{|c|c|c|c|c|}
\hline Baseline characteristics & $\begin{array}{l}\text { Low-dose opioids } \\
\mathrm{N}=115\end{array}$ & $\begin{array}{l}\text { Intermediate-dose opioids } \\
\mathrm{N}=190\end{array}$ & $\begin{array}{l}\text { High-dose opioids } \\
N=117\end{array}$ & $P$ value \\
\hline Age in years, mean (SD) & $67(12)$ & $62(12)$ & $59(11)$ & $<0.001$ \\
\hline Male, n (\%) & $80(70)$ & $149(78)$ & $107(92)$ & $<0.001$ \\
\hline Diabetes, $\mathrm{n}(\%)$ & $18(16)$ & $27(14)$ & $24(21)$ & 0.34 \\
\hline Hypertension, n (\%) & $73(64)$ & $103(54)$ & $65(56)$ & 0.263 \\
\hline Dyslipidaemia, n (\%) & $60(52)$ & $97(51)$ & $72(62)$ & 0.175 \\
\hline $\mathrm{BMI}(\mathrm{IQR} ; \mathrm{n}=271)$ & $26.3(24-30)$ & $27.7(25-30)$ & $28(26-36)$ & 0.014 \\
\hline Current or ex-smoking, $n(\%)(\mathrm{N}=419)$ & $70(62)$ & $136(72)$ & $88(75)$ & 0.069 \\
\hline PVD, n (\%) & $5(4)$ & $6(3)$ & $3(3)$ & 0.74 \\
\hline CVD, n (\%) & $9(8)$ & $9(5)$ & $6(5)$ & 0.504 \\
\hline IHD, n (\%) & $21(18)$ & $26(14)$ & $30(26)$ & 0.031 \\
\hline Previous PCl, n (\%) & $8(7)$ & $16(8)$ & $25(21)$ & 0.001 \\
\hline Previous CABGs, n (\%) & $5(4)$ & $2(1)$ & 0 & 0.024 \\
\hline CCF, n (\%) & $3(3)$ & $5(3)$ & $3(3)$ & 0.999 \\
\hline Creatinine $>120 \mu \mathrm{mol} / \mathrm{L}, \mathrm{n}(\%), \mathrm{N}=421$ & $8(7)$ & $13(7)$ & $11(9)$ & 0.688 \\
\hline $\begin{array}{l}\text { Prehospital duration in minutes (IQR), } \\
\mathrm{N}=406\end{array}$ & $50(42-61)$ & $54(47-65)$ & $64(53-73)$ & $<0.001$ \\
\hline $\begin{array}{l}\text { Triage time in minutes }(\mathrm{IQR}) \text {, } \\
\mathrm{N}=401\end{array}$ & $2(1-5)$ & $3(2-5)$ & $3(2-5)$ & 0.290 \\
\hline $\begin{array}{l}\text { Symptom to intervention time, } \\
\mathrm{N}=406\end{array}$ & $172(126-311)$ & $148(122-199)$ & $165(129-213)$ & 0.181 \\
\hline
\end{tabular}

$\mathrm{N}$ denotes total sample size (or sample size within groups)

$\mathrm{n}$ denotes numbers within each characteristic for each group

BMI, body mass index; CABG, coronary artery bypass graft surgery; CCF, congestive cardiac failure; CVD, cerebrovascular disease; IHD,

ischaemic heart disease; $\mathrm{PCI}$, percutaneous coronary intervention; PVD, peripheral vascular disease.

change in mean CK release. This was presented as the effect of each $1 \mathrm{mg}$ of intravenous morphine equivalent dose on CK release and cardiac MRI infarct size.

Pearson's correlation coefficients were used to evaluate the relationship between opioid dose and cardiac biomarkers and MRI measures of infarct size.

\section{RESULTS}

A total of 422 patients were included in this analysis, with confirmed STEMI undergoing PCI. There were 115 patients within the low-dose opioid group, 190 within the intermediate-dose opioid group and 117 within the highdose opioid group. Baseline characteristics are presented in table 1. Patients receiving high-dose opioids were younger ( 59 years vs 67 years, $\mathrm{p}<0.001$ ) and more likely to be male $(92 \%$ vs $70 \%, \mathrm{p}<0.001)$ than those receiving lowdose opioids. Body mass index (BMI) was also higher in patients receiving high-dose opioids compared with low dose (28 vs $26.3, p=0.014$ ). Patients receiving high-dose opioids were also more likely to have ischaemic heart disease (IHD; $26 \%$ vs $18 \%$, $\mathrm{p}=0.031)$ with prior PCI $(21 \%$ vs $7 \%, \mathrm{p}=0.001$ ) and statistically less likely to have coronary artery bypass graft surgery ( $4 \%$ low dose vs $0 \%$ high dose, $\mathrm{p}=0.024$ ).
In terms of baseline medications, there was a small but statistically significant difference between anticoagulation use between groups at baseline $(4 \%$ in low-dose group vs $0 \%$ in high-dose group, $\mathrm{p}=0.026)$. Otherwise, there was no significant differences in medical therapy at baseline (see online supplementary table 1 ).

In terms of prehospital characteristics, there was no significant difference in heart rate or systolic blood pressure. Importantly, there were no significant differences between oxygen saturations at baseline or administration of supplemental oxygen between the groups.

The median dose of intravenous morphine equivalent analgesia given was $7.5 \mathrm{mg}$ in the low-dose group, $12.5 \mathrm{mg}$ in the intermediate-dose group and $21.5 \mathrm{mg}$ in the highdose group $(\mathrm{p}<0.001$, see online supplementary table 2$)$. The duration from ambulance arrival to hospital admission was significantly longer in the high-dose opioid group compared with the low-dose group (median $64 \mathrm{~min}$ compared with $50 \mathrm{~min}, \mathrm{p}<0.001)$. However, emergency department triage times were not significantly different between the groups (median duration $2 \mathrm{~min}$ vs $3 \mathrm{~min}$ in low-dose and high-dose opioid groups, $\mathrm{p}=0.29$ ). Symptom to intervention times were also not significantly different between the opioid groups (median duration $172 \mathrm{~min}$ in 


\begin{tabular}{|c|c|c|c|c|}
\hline Clinical endpoints & Low-dose opioids & Intermediate-dose opioids & High-dose opioids & $P$ value \\
\hline Median peak CK (U/L) (IQR) n=420; & $1389(665-2756)$ & $1881(1030-3842)$ & $2286(1128-4343)$ & $<0.001$ \\
\hline Median $\mathrm{AUC}_{72} \mathrm{CK}(\mathrm{U} / \mathrm{L})(\mathrm{IQR}) \mathrm{n}=420$ & 44924 (24 264-86 084) & 62501 (33 752-112 361) & 70787 (37 789-107 372 & 0.004 \\
\hline Geometric mean peak CK (U/L) (IQR) $n=420$ & $1339(665-2697)$ & $1808(992-4024)$ & $2208(1097-4447)$ & $<0.001$ \\
\hline Geometric mean $\mathrm{AUC}_{72} \mathrm{CK}(\mathrm{U} / \mathrm{L})(\mathrm{IQR}) \mathrm{n}=417$ & 49021 (26 903-98 716) & 73130 (36 316-133 252) & 80822 (40 135-120 572) & 0.002 \\
\hline Median peak cTnl $(\mu \mathrm{g} / \mathrm{L})(\mathrm{IQR}) \mathrm{n}=390$ & $41(13-110)$ & $75(27-145)$ & $83(30-145)$ & 0.004 \\
\hline Median AUC $_{72} \mathrm{cTnl}(\mu \mathrm{g} / \mathrm{L})(\mathrm{IQR}) \mathrm{n}=388$ & $1392(563-3974)$ & $2397(937-5046)$ & $2757(966-4827)$ & 0.002 \\
\hline Geometric mean peak cTnl ( $\mu \mathrm{g} / \mathrm{L})(\mathrm{IQR}) \mathrm{n}=390$ & $40(12-110)$ & $74(27-148)$ & $81(30-148)$ & 0.004 \\
\hline Geometric mean $\mathrm{AUC}_{72} \mathrm{cTnl}(\mu \mathrm{g} / \mathrm{L})(\mathrm{IQR}) \mathrm{n}=387$ & $1339(602-4024)$ & 2697 (992-5432) & $2981(1097-5432)$ & 0.002 \\
\hline
\end{tabular}

$\mathrm{AUC}_{72}$, area under the biomarker-time curve up to 72 hours post myocardial infarction; CK, creatine kinase; cTnl, cardiac troponin I.

the low opioid dose and $165 \mathrm{~min}$ in the high opioid dose groups, $\mathrm{p}=0.181$ ).

Chest pain score based on numerical rating scale on ambulance arrival to patient was higher in the high-dose opioid group compared with low dose ( 8 vs $6, p<0.001$, see online supplementary table 2 ). It remained higher in the high-dose opioid group compared with low dose at hospital arrival (3 vs $1, \mathrm{p}<0.001$, see online supplementary table 2). Despite higher opioid doses, there was no significant difference in median pain reduction between the groups (four points in all groups, $\mathrm{p}=0.548$ ).
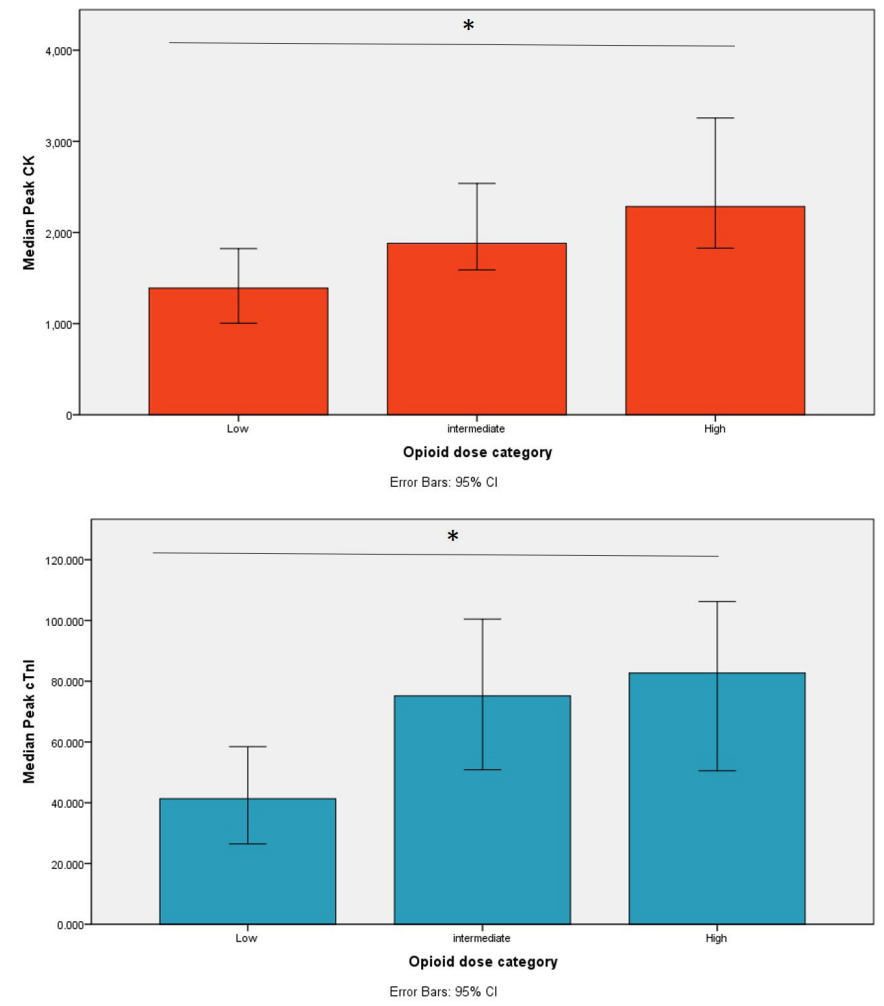

Figure 1 Biomarkers as a surrogate marker of infarct size by opioid dosing category. The top bar graph represents median peak creatine kinase (U/L) and the bottom bar graph median peak cardiac troponin I concentrations $(\mu \mathrm{g} / \mathrm{L})$ in each opioid dosing group (low, intermediate and high). Error bars represent $95 \%$ Cls. *Statistically significant differences between the three groups.
There was a significantly higher proportion of patients with culprit LAD lesions in the high-dose opioid group compared with low dose $(43 \%$ vs $25 \%, \mathrm{p}=0.016$, see online supplementary table 3 ). There was also greater radial access in the high-dose opioid group than low dose ( $44 \%$ vs $28 \%, p=0.022$ ). There was a significantly higher proportion of patients with TIMI 0 or 1 flow pre-PCI in the high dose opioid group compared with low dose (94\% vs $81 \%, p=0.005)$. There was also greater use of thrombus aspiration catheters in the high-dose compared with lowdose opioid group $(59 \%$ vs $30 \%, \mathrm{p}<0.001)$. The door to intervention time was not significantly different between the groups.

There was a significant difference between cardiac biomarkers as a measure of infarct size between highdose, intermediate-dose and low-dose opioids (see table 2). Median peak CK in the high-dose opioid group was $2286 \mathrm{U} / \mathrm{L}$ compared with $1881 \mathrm{U} / \mathrm{L}$ in the intermediate-dose group and $1389 \mathrm{U} / \mathrm{L}$ in the low-dose group $(\mathrm{p}<0.001$; see figure 1$)$. Similarly, median peak cTnI was significantly higher in the high-dose opioid group $(83 \mu \mathrm{g} / \mathrm{L})$ compared with the intermediate-dose and low-dose groups (75 and $41 \mu \mathrm{g} / \mathrm{L}$, respectively, $\mathrm{p}=0.004)$. This was consistent for derived $\mathrm{AUC}_{72}$ and geometric mean values for both biomarkers.

There were no significant differences in clinical endpoints between the three groups except for a small but statistically significant difference in major bleeding (4\% in low-risk group, $5 \%$ in intermediate group and 0 in high-dose group, $\mathrm{p}=0.046$ ). There were no differences in clinical endpoints at 6-month follow-up or between aspirin and oral P2Y12 inhibitor use (including individual P2Y12 inhibitor; see online supplementary table 4).

In the subgroup of patients where infarct size was evaluated with cardiac MRI $(\mathrm{n}=122)$, there was a trend to increased infarct size in the high-dose opioid group (20.7 vs 12.7 in the low-dose group, $\mathrm{p}=0.099$; see online supplementary table 5 ).

The optimal correlation between morphine equivalent opioid dose and cardiac biomarkers based on Pearson correlation coefficient was identified for logarithmic peak CK $(\mathrm{r}=0.194, \mathrm{p}<0.001$; see online supplementary table 6). Therefore, the logarithmic transformed peak 


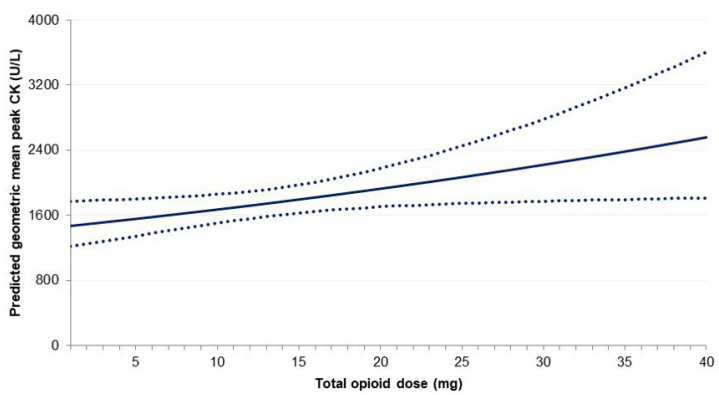

Figure 2 Effect of total opioid dose administration as morphine equivalent dose (in $\mathrm{mg}$ ) on the predicted geometric mean peak creatine kinase (CK; U/L) holding model covariates at their mean value. The total opioid dose (intravenous morphine equivalent dose in $\mathrm{mg}$ ) is represented on the $\mathrm{x}$-axis while predicted geometric mean peak CK $(U / L)$ is represented on the $y$-axis. Model covariates include adjustment for age, sex, diabetes, smoker status, hypertension, hyperlipidaemia, left anterior descending culprit vessel and symptom to intervention time. The dotted lines represent 95\% Cls.

CK was used to evaluate the incremental effect of opioid administration on biomarkers of infarct size using linear regression. The results were then back transformed to generate geometric mean peak CK. The unadjusted estimate from this model demonstrated a $2.5 \%$ increase (95\% CI $1.3 \%$ to $3.7 \%, \mathrm{p}<0.001)$ in geometric mean peak CK for every $1 \mathrm{mg}$ equivalent of intravenous morphine administered. This model was then adjusted for age, sex, diabetes, smoker status, hypertension, hyperlipidaemia, LAD culprit vessel, symptom to intervention time and TIMI flow. A random effects model was used to adjust for hospital of presentation effect. Before adjustment for TIMI flow and hospital effect, for every $1 \mathrm{mg}$ equivalent of intravenous morphine administered, there was an increase in peak CK by $1.4 \%$ (95\% CI $0.2 \%$ to $2.7 \%$, $\mathrm{p}=0.028$ ). After adjustment for hospital effect, opioid dose remained significant with the same effect size of $1.4 \%(95 \%$ CI $0.2 \%$ to $2.7 \%, \mathrm{p}=0.026)$; however, after also adjusting for TIMI flow pre-PCI, opioid dose was no longer significant $(\mathrm{p}=0.084)$. The predicted increase in geometric mean peak CK after holding all other covariates at their mean value is shown in figure 2 .

\section{DISCUSSION}

This is the first study to our knowledge that suggests a dose-dependent signal between opioid use and myocardial infarct size in patients presenting with STEMI. Importantly, despite potential haemodynamic benefits, we did not identify any suggestion that increasing opioid doses were beneficial in terms of clinical, interventional outcomes or measures of infarct size. Alternatively, given that this is a secondary observational analysis, it is possible that increased opioid administration reflects greater ischaemic burden leading to increased severity of chest pain. As such, our findings are hypothesis generating and prospective randomised controlled trials are required to establish causality between opioid dose and myocardial infarct size.

When stratifying patients according to low, intermediate or high doses of opioids, there were significantly higher peak CK and cTnI levels in patients receiving high-dose opioids. There was also a trend towards larger infarct size on cardiac MRI; however, this was not significant and may relate to the relatively small subgroup of patients that had cardiac MRI evaluation of infarct size.

De Waha et al did identify larger infarct size and lower myocardial salvage index in patients with STEMI receiving morphine in a larger sample size. ${ }^{11}$ While our study was not designed to assess clinical outcomes and did not find meaningful differences between the different groups based on opioid dosing, other research groups have demonstrated the impact on clinical outcomes with findings suggestive of increased mortality, longer hospital stay and greater complications. ${ }^{2}{ }^{12}$ However, there are conflicting results from retrospective, observational studies in this respect. ${ }^{3513}$

Interestingly, patients receiving high-dose opioids were more likely to have TIMI 0 or 1 flow pre-PCI and the greater use of thrombus aspiration catheters, suggesting a greater thrombus burden in these patients. This may relate to delayed action of P2Y12 inhibitors prior to emergent coronary angiography in the context of gastroparesis secondary to high-dose opioids. Alternatively, TIMI 0 or 1 flow may have prompted greater opioid administration due to chest pain due to myocardial ischaemia in the myocardial territory supplied by culprit vessel. Bellandi et al identified lower ST-segment resolution rates and lower pre-PCI TIMI flow rates in patients treated with morphine. ${ }^{4}$ Delayed antiplatelet effects have been demonstrated in multiple biochemical studies evaluating oral P2Y12 inhibitors in combination with morphine or fentanyl. ${ }^{14-18}$ Hence, these studies provide biochemical evidence of delayed peak plasma concentrations of P2Y12 inhibitors or their metabolites as well as higher on treatment platelet reactivity particularly in the first 2 hours when patients undergo primary PCI.

When evaluating the group receiving high-dose opioids, there were some important baseline differences. These patients were younger, more likely to be male, had an increased BMI and were more likely to have IHD. Additionally, prehospital duration was significantly longer in the high opioid dose group which may reflect greater distance travelled from first medical contact to hospital arrival. Higher opioid doses may have been required during the longer prehospital duration if the culprit vessel remained occluded until definitive coronary intervention was performed. Predictably, patients in the high opioid dose group had higher pain scores on ambulance arrival, however despite greater doses of opioids, had higher pain scores on arrival to hospital. Despite the variation in the dose of opioids, the average pain reduction was not significantly different between the three groups. Most patients received morphine with approximately $10 \%$ across the groups receiving fentanyl. Interestingly, use of nitroglycerin 
was relatively low at approximately $20 \%$ across the groups and did not differ significantly. Use of other analgesics such as methoxyflurane was very low (4 patients out of 422). This suggests that while it is standard care, higher doses of opioids were generally ineffective in controlling severe ischaemic chest pain. As such, alternative analgesic agents may be beneficial in this patient cohort by avoiding the interaction with P2Y12 inhibitors and potentially providing more effective pain relief.

Several strategies have been trialled to mitigate the opioid-P2Y12 interaction. The use of methylnaltrexone, a peripheral opioid receptor antagonist, was not shown to improve bioavailability of ticagrelor. ${ }^{19}$ On the other hand, metoclopramide which increases gastrointestinal motility was effective in improving the antiplatelet effects of ticagrelor in the presence of morphine.$^{20}$ Alternatively, bridging with cangrelor, an intravenous P2Y12 inhibitor may be an effective strategy for tackling delayed bioavailability of oral P2Y12 inhibitors. $^{21}$

After adjustment for multiple comorbidities and hospital effect, each $1 \mathrm{mg}$ of morphine equivalent was associated with a $1.4 \%$ increase in peak CK; however, this was no longer statistically significant after adjusting for TIMI flow pre-PCI. All model results were included to enable balanced analysis of the results. However, adjustment for TIMI flow is challenging as poor TIMI flow pre-PCI may be due to higher opioid doses given to patients or may be a reflection of increased pain due to a completely occluded artery for which higher opioid doses were given. As such, statistical attempts at delineating the independent effect of TIMI flow and opioid dose on biomarkers of infarct size are flawed and we believe that a randomised controlled trial is the only accurate way of determining this.

\section{Limitations}

There are several limitations of our study. Given that the study is a post-hoc analysis of a randomised controlled trial, the lack of randomisation related to allocation of opioid therapy may be responsible for an imbalance of confounding factors between the groups. For example, it is possible that the association identified between opioid dose and infarct size in this study may be due to patients with larger infarct sizes experiencing more pain and therefore receiving more opioids. Therefore, this analysis is exploratory and hypothesis generating. The study is also affected by limitations of the original study. These include limited application of cardiac MRI to a subgroup population, lack of central core laboratory for assessment of biomarkers and exclusion of $8.2 \%$ of patients due to incomplete CTnI assays and $0.5 \%$ of patients due to incomplete CK assays. The assays used to evaluate biomarkers were not standardised between the different hospitals but we have adjusted for hospital effect and found no significant effect on the association between opioid dose and cardiac biomarkers of infarct size. Furthermore, while there is excellent record keeping for administration of opioid dosing in the prehospital setting, we do not have data relating to in-hospital opioid administration. Given that patients spend minimum time in emergency departments prior to emergent coronary angiography, we do not expect that patients received large doses of opioids in hospital and therefore do not expect this to alter our findings.

Finally, while the proposed mechanism of increased infarct size is the delayed onset and activity of oral P2Y12 inhibitors, we do not have information on timing of oral P2Y12 inhibitors during index admission, loading dose used or results of platelet function testing results to support this mechanism. However, previous biochemical studies have demonstrated that opioid analgesia delays the onset of action of all oral P2Y12 inhibitors in patients with acute myocardial infarction. ${ }^{182}$

\section{CONCLUSIONS}

Our study did not demonstrate any benefit of higher opioid doses in terms of measures of infarct size in STEMI but rather a signal towards a dose-dependent increase in CK release as a marker of infarct size with increasing opioid dose in patients with STEMI. This may be due to opioids interacting with oral P2Y12 inhibitors or may reflect greater opioid administration to patients with a greater burden of ischaemia prior to coronary intervention. Prospective clinical studies are required to accurately differentiate between these two explanations. In the meantime, we believe that future research needs to focus on identifying strategies to mitigate the opioid-P2Y12 inhibitor interaction and alternative analgesia to treat ischaemic chest pain.

\section{Author affiliations \\ ${ }^{1}$ Department of Cardiology, Alfred Hospital, Melbourne, Victoria, Australia \\ ${ }^{2}$ Central Clinical School, Monash University, Melbourne, Victoria, Australia ${ }^{3}$ Atherothrombosis and Vascular Biology, Baker Heart Research Institute, Melbourne, Victoria, Australia \\ ${ }^{4}$ Department of Epidemiology and Preventive Medicine, Monash University, Melbourne, Victoria, Australia \\ ${ }^{5}$ Research \& Evaluation, Ambulance Victoria, Doncaster, Victoria, Australia ${ }^{6}$ Intensive Care Unit, Alfred Hospital, Melbourne, Victoria, Australia \\ ${ }^{7}$ Heart Centre, Baker Heart Research Institute, Melbourne, Victoria, Australia}

Acknowledgements The authors are grateful to the assistance of all the paramedics and hospital staff that contributed to the AVOID trial.

Contributors DS conceived and designed this research analysis. HF, ZN and DS acquired the data and performed the statistical analysis. KP, SB, MS, JB, PC, AE, AT, DKM, KS and DS handled funding and supervision of the AVOID trial and critical review of the current manuscript for key intellectual content which was drafted by HF and DS.

Funding The AVOID study was funded by grants from Alfred Foundation, FALCK foundation and Paramedics Australia.

Competing interests $\mathrm{ZN}$ is funded by a National Health and Medical Research Council Early Career Fellowship (\#1146809). DS is funded by National Heart Foundation Fellowship and Viertel Foundation Grant.

Patient consent for publication Not required.

Ethics approval Alfred Health Human Research Ethics Committee.

Provenance and peer review Not commissioned; externally peer reviewed.

Data availability statement Data may be obtained from a third party and are not publicly available. Deidentified data generated from the AVOID study requires ethics approval prior to being made available.

Open access This is an open access article distributed in accordance with the Creative Commons Attribution Non Commercial (CC BY-NC 4.0) license, which 
permits others to distribute, remix, adapt, build upon this work non-commercially, and license their derivative works on different terms, provided the original work is properly cited, appropriate credit is given, any changes made indicated, and the use is non-commercial. See: http://creativecommons.org/licenses/by-nc/4.0/.

ORCID iDs

Himawan Fernando http://orcid.org/0000-0002-4775-6708

Dion Stub http://orcid.org/0000-0001-8686-2709

\section{REFERENCES}

1 Rouby JJ, Eurin B, Glaser P, et al. Hemodynamic and metabolic effects of morphine in the critically ill. Circulation 1981;64:53-9.

2 Meine TJ, Roe MT, Chen AY, et al. Association of intravenous morphine use and outcomes in acute coronary syndromes: results from the crusade quality improvement initiative. Am Heart $J$ 2005;149:1043-9.

3 lakobishvili Z, Porter A, Battler A, et al. Effect of narcotic treatment on outcomes of acute coronary syndromes. Am J Cardiol 2010;105:912-6.

4 Bellandi B, Zocchi C, Xanthopoulou I, et al. Morphine use and myocardial reperfusion in patients with acute myocardial infarction treated with primary PCI. Int J Cardiol 2016;221:567-71.

5 Koh JQS, Fernando H, Peter K, et al. Opioids and ST elevation myocardial infarction: a systematic review. Heart Lung Circ 2019;28:697-706.

6 Nehme Z, Stub D, Bernard S, et al. Effect of supplemental oxygen exposure on myocardial injury in ST-elevation myocardial infarction. Heart 2016;102:444-51.

7 Stub D, Smith K, Bernard S, et al. Air versus oxygen in ST-segmentelevation myocardial infarction. Circulation 2015;131:2143-50.

8 Stub D, Smith K, Bernard S, et al. A randomized controlled trial of oxygen therapy in acute myocarDial infarction air verses oxygen in myocarDial infarction study (avoid study). Am Heart $J$ 2012;163:339-45.

9 White IR, Royston P, Wood AM. Multiple imputation using chained equations: issues and guidance for practice. Stat Med 2011;30:377-99.

10 Rubin DB, Schenker N. Multiple imputation in health-care databases: an overview and some applications. Stat Med 1991;10:585-98.
11 de Waha S, Eitel I, Desch S, et al. Intravenous morphine administration and reperfusion success in ST-elevation myocardial infarction: insights from cardiac magnetic resonance imaging. Clin Res Cardiol 2015;104:727-34.

12 McCarthy CP, Bhambhani V, Pomerantsev E, et al. In-Hospital outcomes in invasively managed acute myocardial infarction patients who receive morphine. J Interv Cardiol 2018;31:150-8.

13 Bonin M, Mewton N, Roubille F, et al. Effect and safety of morphine use in acute anterior ST-segment elevation myocardial infarction. $J$ Am Heart Assoc 2018;7:e006833.

14 Hobl E-L, Reiter B, Schoergenhofer C, et al. Morphine interaction with prasugrel: a double-blind, cross-over trial in healthy volunteers. Clin Res Cardiol 2016;105:349-55.

15 Hobl E-L, Stimpfl T, Ebner J, et al. Morphine decreases clopidogrel concentrations and effects: a randomized, double-blind, placebocontrolled trial. J Am Coll Cardiol 2014;63:630-5.

16 Hobl E-L, Reiter B, Schoergenhofer C, et al. Morphine decreases ticagrelor concentrations but not its antiplatelet effects: a randomized trial in healthy volunteers. Eur J Clin Invest 2016;46:7-14.

17 Kubica J, Adamski P, Ostrowska M, et al. Morphine delays and attenuates ticagrelor exposure and action in patients with myocardial infarction: the randomized, double-blind, placebo-controlled impression trial. Eur Heart J 2016;37:245-52.

18 Ibrahim K, Shah R, Goli RR, et al. Fentanyl delays the platelet inhibition effects of oral ticagrelor: full report of the PACIFY randomized clinical trial. Thromb Haemost 2018;118:1409-18.

19 Franchi F, Rollini F, Park Y, et al. Effects of the peripheral opioid receptor antagonist methylnaltrexone on the $\mathrm{pK}$ and $\mathrm{PD}$ profiles of ticagrelor in patients with coronary artery disease treated with morphine. JACC Cardiovasc Interv 2019.

20 Sikora J, Niezgoda P, Barańska M, et al. Metoclopramide administration as a strategy to overcome MORPHine-ticagrelOr interaction in patients with unstable angina PectorlS-The metamorphosis trial. Thromb Haemost 2018;118:2126-33.

21 Franchi F, Rollini F, Rivas A, et al. Platelet inhibition with cangrelor and crushed ticagrelor in patients with ST-segment-elevation myocardial infarction undergoing primary percutaneous coronary intervention. Circulation 2019;139:1661-70.

22 Kubica J, Adamski P, Ostrowska M, et al. Influence of morphine on pharmacokinetics and pharmacodynamics of ticagrelor in patients with acute myocardial infarction (impression): study protocol for a randomized controlled trial. Trials 2015;16:1-6. 